\title{
Cooperative and anticooperative effects in resonance assisted hydrogen bonds in merged structures of malondialdehyde
}

\author{
Eduardo Romero-Montalvo ${ }^{\mathrm{a}}$, José Manuel Guevara-Vela ${ }^{\mathrm{b}}$, Aurora Costales ${ }^{\mathrm{b}}$, Ángel Martín \\ Pendás ${ }^{\mathrm{b}}$, Tomás Rocha Rinzaa,* \\ ${ }^{a}$ Institute of Chemistry, National Autonomous University of Mexico, Circuito Exterior, Ciudad \\ Universitaria, Delegación Coyoacán C.P. 04510, Mexico City, Mexico. \\ ${ }^{b}$ Departament of Analytical and Physical Chemistry, University of Oviedo, E-33006, Oviedo, Spain.
}

\begin{abstract}
We analyzed non-additive effects in resonance assisted hydrogen bonds (RAHB) in different $\beta$-enolones, which are archetypal compounds of this type of interactions. For this purpose, we used $(i)$ potential energy curves to compute the formation energy, $\Delta E_{\text {form }}^{\text {RAHB }}$, of the RAHBs of interest in different circumstances along with (ii) tools offered by quantum chemical topology, namely, the quantum theory of atoms in molecules (QTAIM) and the Interacting Quantum Atoms (IQA) electronic energy partition. We established the effect that a given $\mathrm{H}$-bond exert over $\Delta E_{\text {form }}^{\mathrm{RAHB}}$ associated to another RAHB, determining in this way the cooperativity or anticooperativity of these interactions. The mesomeric structures and the QTAIM delocalisation indices are consistent with the determined cooperative or anticooperative character of two given RAHBs. The HB cooperativity and anticooperativity studied herein are directly reflected in the IQA interaction energy $E_{\mathrm{int}}^{\mathrm{O} \cdots \mathrm{H}}$, but they are modulated by the surrounding hydrocarbon chain. The IQA decomposition of $\Delta E_{\text {coop }}$, a measure of the cooperativity between a pair of interacting RAHBs, indicates that the analyzed H-bond cooperative/anticooperative effects are associated with greater/smaller $(i)$ strenghtenings of the pseudo-bicyclic structure of the compounds of interest and ( $i i$ ) electron localisations with its corresponding changes in the intra and intermolecular exchange-correlation contributions to $\Delta E_{\text {form }}^{\mathrm{RAHB}}$. Overall, we expect that this investigation will provide valuable insights of the interplay present among hydrogen bonded atoms and the $\pi$ system in RAHBs contributing in this way to the understanding of the general features of H-bonds.
\end{abstract}

Keywords:

Resonance assisted hydrogen bonds, H-bond cooperative and anticooperative effects, Potential energy curves, Quantum theory of atoms in molecules, Interacting quantum atoms

*To whom correspondence should be addressed: tomasrocharinza@gmail.com 


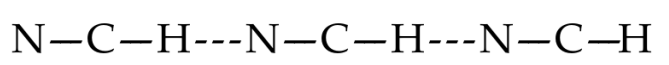

(a)

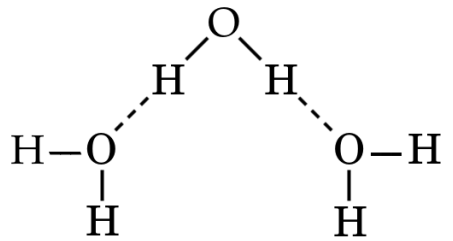

(b)

Figure 1: (a) Hydrogen bond cooperative effects in the linear HCN trimer: both H-bonds strengthen each other. (b) Water molecule acting as a double hydrogen bond donor: the H-bonds are reciprocally weakened.

\section{Introduction}

Since its discovery in the $1920 \mathrm{~s}^{[1]}$ many different experimental and theoretical investigations have been dedicated to the hydrogen bond (HB). The importance of this interaction resides not only on its effects on diverse systems in chemistry and biology but also on its complexity. For instance, the energetics of an $\mathrm{O}-\mathrm{H} \cdots \mathrm{O}$ H-bond might lie in the range of $1-30 \mathrm{kcal} / \mathrm{mol}^{[2]}$. Further problems in the characterization of HBs arise from the dissimilar proportions of their covalent, electrostatic and dispersion contributions ${ }^{[3]}$. This situation has lead to the coinage of the term "H-bond puzzle" to indicate the inability to fully understand the nature of hydrogen bonds ${ }^{[4]}$. Indeed, the intricated character of HB makes it very versatile and ubiquitous in many processes: from giving water its extraordinary features ${ }^{[5]}$ to the HB involvement in complicated biochemical reactions ${ }^{[6]}$ among other phenomena.

Part of the difficulty in the understanding of HB originates in its non-additivity. Apart from the electrostatic component in non-covalent interactions, the distinct contributions to the HB formation energy are non-additive ${ }^{[7]}$, i.e., the value of the component of the interaction energy of two species is affected by the presence of a third electronic system, which leads to cooperative and anticooperative effects of HB. The former occurs when hydrogen bonds strengthen each other whereas the latter involves a mutual weakening of the interactions. For example, the HBs in a linear structure of the type $\mathrm{BH} \cdots \mathrm{BH} \cdots \mathrm{BH}$, like that shown in Figure 1(a) display cooperative effects ${ }^{[8]}$ as opposed to those present in a double hydrogen bond donor (Figure 1(b)) which exhibit HB anticooperativity. The charge transfer leading to the referred non-additive effects takes place through $\sigma$ bonds and hence it is called " $\sigma$-bond" cooperativity or anticooperativity. ${ }^{[3]}$

In addition to these $\sigma$-bond non-additive effects, conjugated systems might also influence 


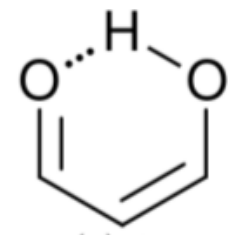

(a) 1

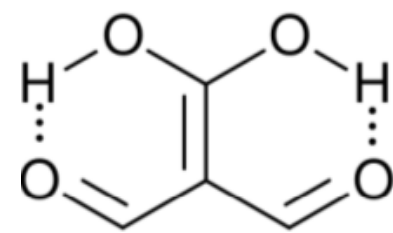

(c) 3

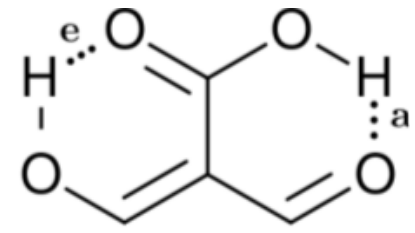

(b) 2

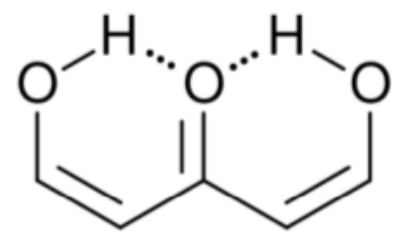

(d) 4

Figure 2: Systems addressed in this study. X-ray crystallography results suggest that the H-bonds within compound $\mathbf{2}$ strenghten each other as opposed to systems $\mathbf{3}$ and $\mathbf{4}$ which exhibit H-bond anticooperativity ${ }^{[18]}$.

HBs, for example in $\beta$-diketones ${ }^{[9]}$ shown in Figure 2. These H-bonds are called Resonance Assisted Hydrogen Bonds (RAHB) and they were introduced as "the interplay between hydrogen bond and heterodienes (or more generally heteroconjugated systems) leading to a strengthening of the hydrogen bond itself". ${ }^{[9]}$ Figure 2 (a) shows this interaction between the hydrogen bond and the $\pi$ system within malondialdehyde, $\mathrm{CHO}-\mathrm{CH}=\mathrm{CH}-\mathrm{OH}$. Resonance assisted hydrogen bonds have been of considerable interest in physical chemistry, a situation which has lead to numerous theoretical ${ }^{[10-12]}$ and experimental ${ }^{[13,14]}$ reports concerned with the nature of these HBs. In addition, the RAHB concept has been used to better understand intermolecular interactions in relevan systems in chemistry and biology, like those present in DNA ${ }^{[15-17]}$ and proteins ${ }^{[3]}$.

The HB cooperative and anticooperative effects that might occur due to an adjacent $\pi$ system, as in Figure 2 (b)-(d), have been however relatively unexplored ${ }^{[18-21]}$. We chose these systems to study $\pi$-bond HB cooperativity and anticooperativity, because it has been suggested that compound 2 presents HB cooperative effects whereas it occurs the opposite for systems 3 and 4 , i.e. the H-bonds within these systems weaken each other ${ }^{[18]}$. Thus, this work deals with the examination of the hydrogen bond $\pi$ non-additive effects in RAHBs along with the reciprocal action between these HBs and the accompanying conjugated system by considering molecules 1-4 in Figure 2 as archetypal models. For this aim, we calculated potential energy curves to determine the RAHB formation energy, $\Delta E_{\mathrm{form}}^{\mathrm{RAHB}}$, of the intramolecular $\mathrm{H}$ bonds in Figure 2 in distinct circumstances which enabled us to assess the nature of the $\pi$ non-additive effects in these systems. In addition, we used tools offered by quantum chemical topology to get further insights in the studied RAHBs. More specifically, we employed the Interacting Quantum Atoms (IQA) energy partition as well as the Quantum Theory of Atoms 
in Molecules (QTAIM) to analyze the interplay between a given RAHB and its surrounding $\pi$ bonds as well as the resultant HB cooperative or anticooperative effects. We have chosen these methods of wavefunction analyses for this investigation due to its successful use in the study of different non-additive interactions such as those in H-bond within small water clusters $^{[22,23]}$, back-bonding in transition metal carbonyl complexes ${ }^{[24,25]}$, or in $n \rightarrow \pi^{\star}$ transitions in electronic excited states ${ }^{[26]}$. Altogether, our results provide an assessment of the interaction of an RAHB and its neighboring cojugated system on top of HB $\pi$ cooperative and anticooperative effects. We expect that the results presented in this investigation will prove useful in a further understanding of the imporant non-additive effects within resonance assisted hydrogen bonds.

\section{Methods}

The wavefunction analyses presented in this work are based on a partition of the real space into disjoint basins in accordance with the QTAIM ${ }^{[27]}$, an approach founded on the topology of the charge distribution $\varrho_{1}(\mathbf{r})$, which is invariant under orbital rotations because it is the expectation value of a Dirac observable, i.e., $\varrho_{1}(\mathbf{r})=\left\langle\sum_{i} \delta\left(\mathbf{r}-\mathbf{r}_{i}\right)\right\rangle^{[28]}$. The QTAIM basins, denoted generally as $\Omega$, are proper open subsystems for which one can compute the expectation values of quantum mechanical operators such as the Hamiltonian, the kinetic energy, and the number operators. ${ }^{27]}$ The QTAIM also defines localisation and delocalisation indices (LI and DI respectively) in terms of the integration of the Fermi and Coulomb correlation holes over the QTAIM atoms. ${ }^{[29]}$. The LI and DI descriptors are useful to examine electron sharing, i.e. covalency, in different chemical bonding scenarios. The sum of the atomic properties, $\langle O\rangle_{\Omega}$, throughout the whole of the system, yields the expectation value of the corresponding observable for the complete molecule or molecular cluster under consideration. Since the QTAIM is completely built on $\varrho_{1}(\mathbf{r})$, it can be used to address on equal footing a wide variety of chemical compounds and processess, such as metal-ligand bonding ${ }^{[24]}$, interactions within a molecular cluster ${ }^{[30]}, \pi$-electron delocalised ${ }^{[31]}$ and H-bonded systems ${ }^{[12,32]}$, being the two last-mentioned examples of particular interest for this work.

In a similar fashion to QTAIM, the IQA energy partition is based in scalar fields derived from the wavefunction, more specifically, the first order reduced density matrix, $\varrho_{1}\left(\mathbf{r}_{1}, \mathbf{r}_{1}^{\prime}\right)$ and the pair density, $\varrho_{2}\left(\mathbf{r}_{1}, \mathbf{r}_{2}\right)$. The availability of $\varrho_{1}\left(\mathbf{r}_{1}, \mathbf{r}_{1}^{\prime}\right)$ and $\varrho_{2}\left(\mathbf{r}_{1}, \mathbf{r}_{2}\right)$, along with a sectioning of the real space, e.g., the one defined by QTAIM, allows to dissect the electronic energy of a system as 


$$
E=\sum_{\mathrm{A}} E_{\mathrm{net}}^{\mathrm{A}}+\frac{1}{2} \sum_{\mathrm{A}} \sum_{\mathrm{B} \neq \mathrm{A}} E_{\mathrm{int}}^{\mathrm{AB}},
$$

wherein $E_{\text {net }}^{\mathrm{A}}$ denotes the net energy of basin A whereas $E_{\mathrm{int}}^{\mathrm{AB}}$ indicates the interaction energy between atoms $\mathrm{A}$ and $\mathrm{B}$. The pairwise nature of the potential energy operator in the electron Hamiltonian allows to further divide the IQA net and interaction energies as

$$
\begin{aligned}
& E_{\mathrm{net}}^{\mathrm{A}}=T^{\mathrm{A}}+V_{\mathrm{ne}}^{\mathrm{AA}}+V_{\mathrm{ne}}^{\mathrm{AA}}, \\
& E_{\mathrm{int}}^{\mathrm{AB}}=V_{\mathrm{nn}}^{\mathrm{AB}}+V_{\mathrm{ee}}^{\mathrm{AB}} V_{\mathrm{ne}}^{\mathrm{AB}}+V_{\mathrm{ne}}^{\mathrm{BA}} .
\end{aligned}
$$

$T^{\mathrm{A}}$ in equation (2) represents the kinetic energy of basin $\mathrm{A}$ and by letting $\sigma$ and $\tau$ denote either nuclei or electrons, then $V_{\sigma \tau}^{\mathrm{AB}}$ designates the contribution to the potential energy from the interaction of $\sigma$ contained in atom A with $\tau$ within basin B. Additionally, the LHS of equation (1) can be expressed in terms of IQA additive energies,

$$
E=\sum_{\mathrm{A}}\left(E_{\mathrm{net}}^{\mathrm{A}}+\frac{1}{2} \sum_{\mathrm{B} \neq \mathrm{A}} E_{\mathrm{int}}^{\mathrm{AB}}\right)=\sum_{\mathrm{A}} E_{\mathrm{add}}^{\mathrm{A}},
$$

whose sum yields the total electronic energy in a similar fashion to the QTAIM atomic ener$\operatorname{gies}^{[27]}$.

The separation of $\rho_{2}\left(\mathbf{r}_{1}, \mathbf{r}_{2}\right)$ in its coulombic $(\mathrm{J})$ and exchange-correlation (xc) contributions

$$
\varrho_{2}\left(\mathbf{r}_{1}, \mathbf{r}_{2}\right)=\varrho_{2}^{\mathrm{J}}\left(\mathbf{r}_{1}, \mathbf{r}_{2}\right)+\varrho_{2}^{\mathrm{xc}}\left(\mathbf{r}_{1}, \mathbf{r}_{2}\right),
$$

may be utilized to define a classical, that is, electrostatic,

$$
V_{\mathrm{cl}}^{\mathrm{AB}}=V_{\mathrm{J}}^{\mathrm{AB}}+V_{\mathrm{ne}}^{\mathrm{AB}}+V_{\mathrm{ne}}^{\mathrm{BA}}+V_{\mathrm{nn}}^{\mathrm{AB}},
$$

component of $E_{\mathrm{int}}^{\mathrm{AB}}$, along with a quantum, i.e., exchange-correlation contribution which fulfil the relation

$$
E_{\mathrm{int}}^{\mathrm{AB}}=V_{\mathrm{cl}}^{\mathrm{AB}}+V_{\mathrm{xc}}^{\mathrm{AB}}
$$

The division of $E_{\mathrm{int}}^{\mathrm{AB}}$ in equation (7) allows to assess the covalent or ionic character of a given interaction $\mathrm{A}-\mathrm{B}$, which makes the IQA energy partition a powerful tool for the analysis of distinct chemical interactions. 


\section{Computational details}

Because the examined RAHBs are intramolecular, it is not possible to take appart the fragments linked by an H-bond in order to analyse the changes in the system after the dissociation of the HB. Therefore, we computed potential energy curves as a function of the rotation of the $\mathrm{C}-\mathrm{OH}$ bond, as illustrated in
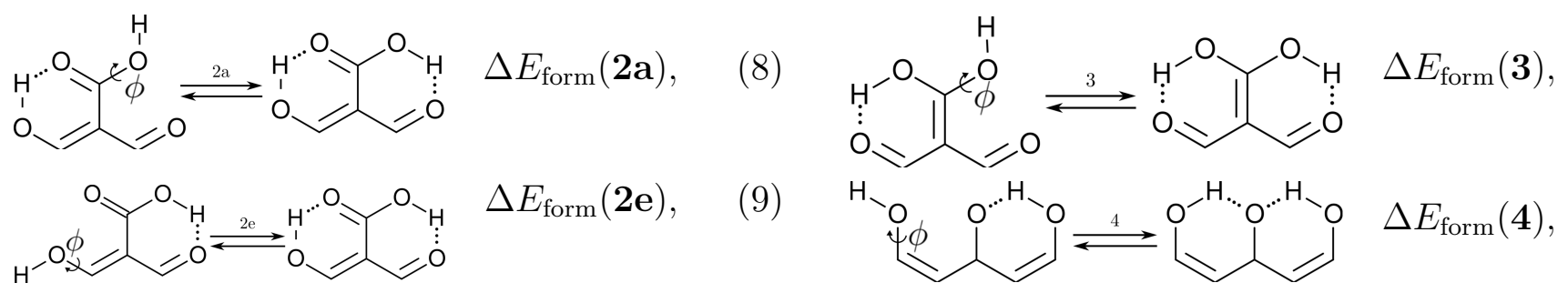

and
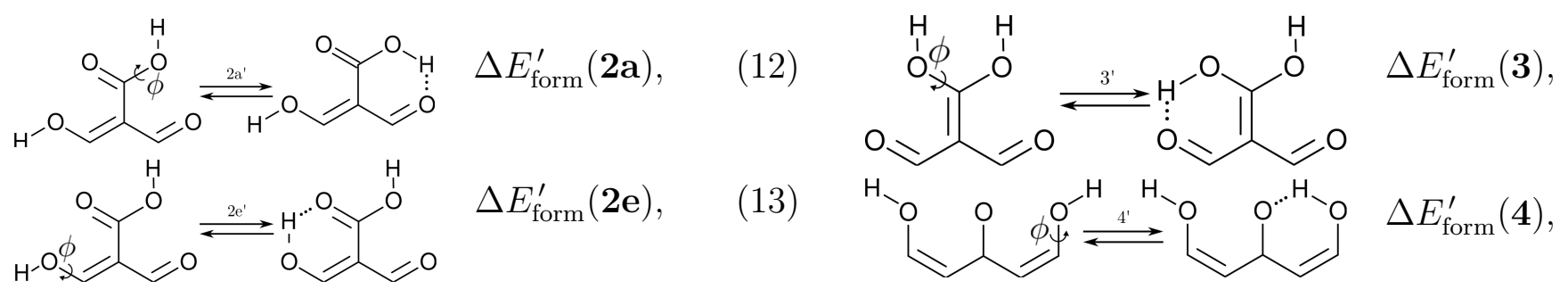

in order to determine the formation energy of each RAHB in presence and absence of the accompanying H-bond in the molecule. The potential energy curves were computed with the MP2 ${ }^{[33]}$ method in its efficient RIJCOSX variant ${ }^{[34]}$ together with the aug-cc-pVTZ basis functions ${ }^{[35]}$ as implemented in the ORCA program package ${ }^{[36]}$. We chose to use the MP2/aug-ccpVTZ approximation, since $\mathrm{O}-\mathrm{H} \cdots \mathrm{O}$ hydrogen-bonded systems are well accounted by second order Møller-Plesset perturbation theory and augmented Dunning basis sets ${ }^{[37]}$.

Afterwards, we carried out the IQA and QTAIM wavefunction analysis based on HF density functions which were computed with the Gamess-Us ${ }^{[38]}$ software. The use of HF wavefunctions is justified, because this approach was recently used to successfully study the H-bond cooperative effects within small water clusters ${ }^{[22]}$ and the nature of the resonance assisted hydrogen bonds ${ }^{[39]}$. Furthermore, the RAHB formation energies examined in this work are considerably larger in magnitude than those within these $\mathrm{H}_{2} \mathrm{O}$ systems. Besides, HF and MP2 approximations give a similar description of the potential energy curves for determining the RAHB formation energy of $\mathbf{1}$ and those in equations (8)-(11) as shown in Figures S1 and S2 in the Supporting Information (SI).

The QTAIM and IQA analyses were carried out with the AIMALL ${ }^{[40]}$ and PROMOLDEN ${ }^{[41]}$ 
programs respectively. In addition, we used the software packages GNuplot ${ }^{[42]}$ and AvoGADRO $^{[43,44]}$ to visualize data and chemical structures.

\section{Results and discussion}

\subsection{Potential energy curves}

We assessed RAHB cooperative and anticooperative effects of the systems addressed in this work by comparing the formation energy of the hydrogen bonds under consideration, with (equations (8)-(11)) and without (expressions (12)-(15)) the accompanying RAHB in the molecule. That is to say, we contrast the quantities $\Delta E_{\text {form }}(\mathbf{2} \mathbf{a}), \Delta E_{\text {form }}^{\prime}(\mathbf{2} \mathbf{e}) ; \Delta E_{\text {form }}(\mathbf{2} \mathbf{e})$, $\Delta E_{\text {form }}^{\prime}(\mathbf{2 e}) ;$ etc.

Figure 3 shows the corresponding potential energy curves for the processes (8)-(15). The zero of every potential energy curve corresponds to the minimum in which $\phi$ is close to $180^{\circ}$, that is, where the RAHB is dissociated. This allows us to read directly $\Delta E_{\text {form }}^{\mathrm{RAHB}}$ at $\phi=0$. We note that generally, the magnitude of the RAHBs formation energies are considerably larger than in other uncharged $\mathrm{O}-\mathrm{H} \cdots \mathrm{O}$ systems, e.g. water clusters, which allows to establish the strong effect of the $\pi$ system over the H-bond energetics. We point out that the scanning of the dihedral angle for system 3 computed with the RIJCOSX-MP2/aug-cc-pVTZ approximation is accompanied by the intramolecular proton transfer shown in Figure 4. This process entails the transformation of compound $\mathbf{3}$ into $\mathbf{2}$. For this reason, we built the corresponding potential energy curve in Figure S2 in the SI by constraining the bond length of the dissociated $\mathrm{O}-\mathrm{H}$ bond to $1.02 \AA$, i.e., its distance in the local minimum at $\phi=0$. The referred intramolecular Brønsted-Lowrry reaction might be related to the fact that so far it has not been possible to synthesize cyrstals with moiety 3 in their structure. ${ }^{[18]}$

We find that the formation energy of H-bond $\mathbf{2 a}$ is larger in the presence of HB $\mathbf{2 e}$ (Figure 3-2a) and vice versa (Figure 3-2b). That is to say, these H-bonds strengthen each other and present cooperative effects throughout the $\pi$ system. The opposite situation is observed in the H-bonds within systems 3 and 4: these interactions weaken one another and present $\pi$ anticooperative effects. These results are in concordance with the experimental evidence ${ }^{[18]}$ and the mesomeric structures of Figure 5. The resonance structure of compound 2 suggest that the RAHBs $\mathbf{2 a}$ and $\mathbf{2} \mathbf{b}$ strengthen each other, whereas in systems $\mathbf{3}$ and $\mathbf{4}$ both RAHBs compete for the $\pi$ electrons within the system. Besides, the central carbonyl in 4 is a double H-bond acceptor which may also induce HB anticooperative effects ${ }^{[3,23]}$.

In order to analyse more thoroughly these non-additive effects, we can consider the differences between the formation energies of the RAHBs within a molecule in presence of the 

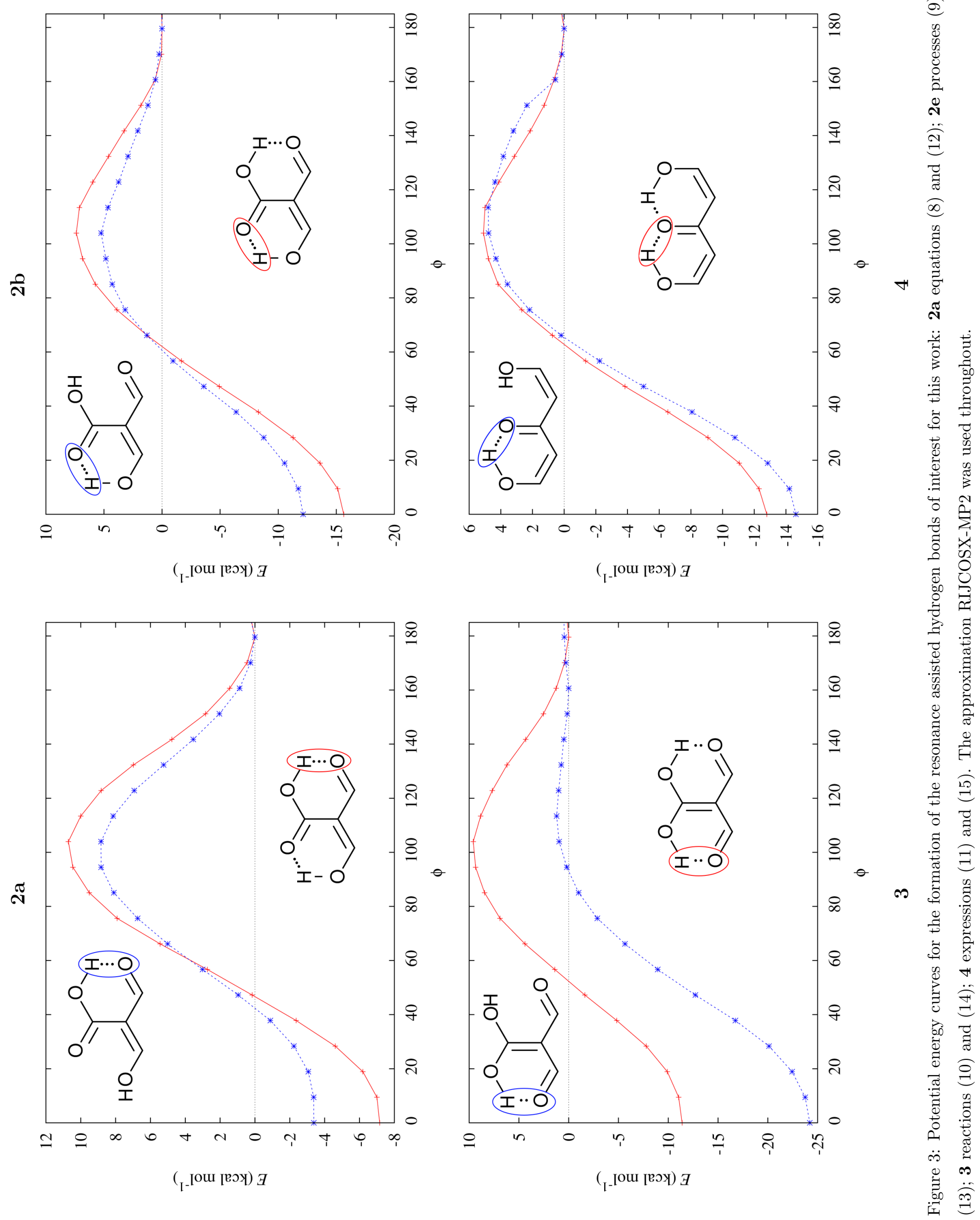


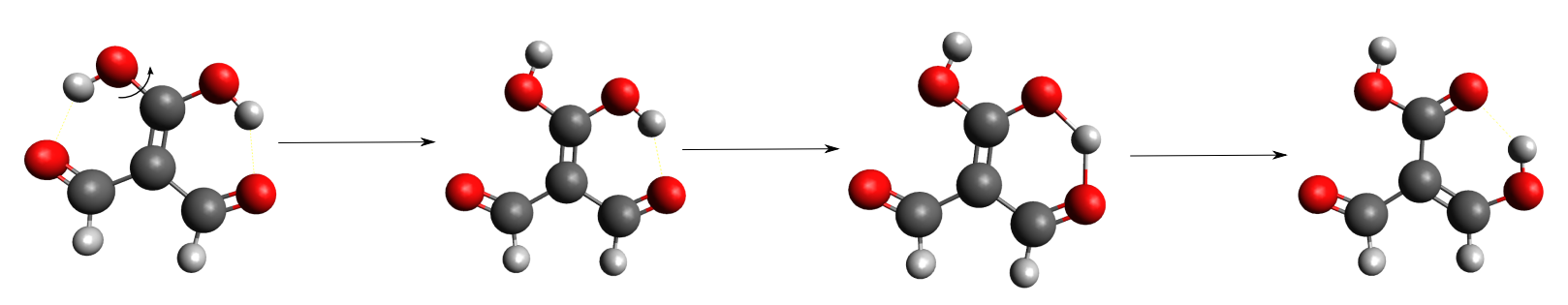

Figure 4: Proton transfer occurred in the scanning of the dihedral angle of system 3 using the approximation RIJCOSX-MP2/aug-cc-pVTZ.

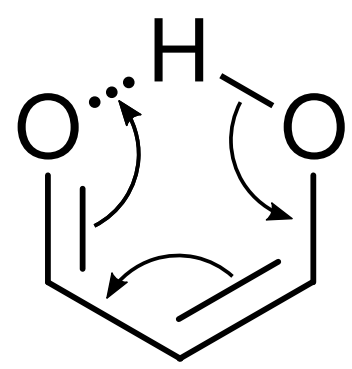

(a) 1

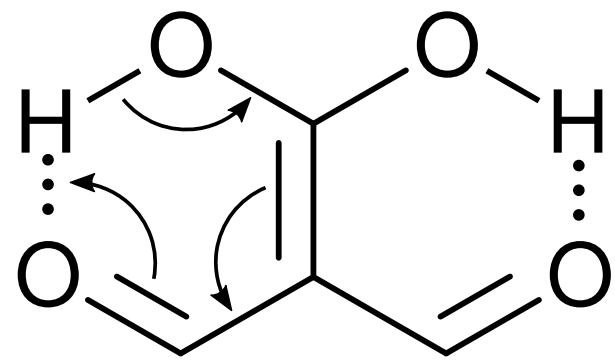

(c) 3

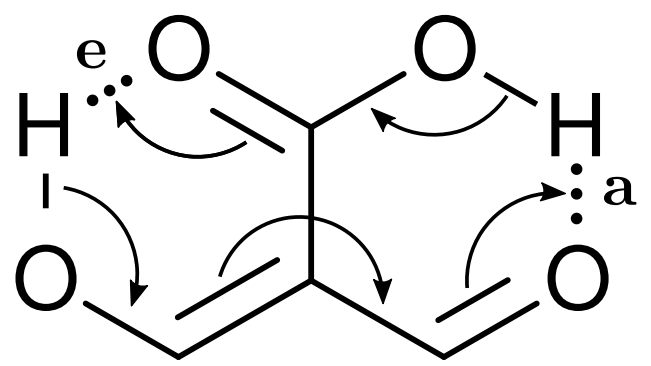

(b) 2

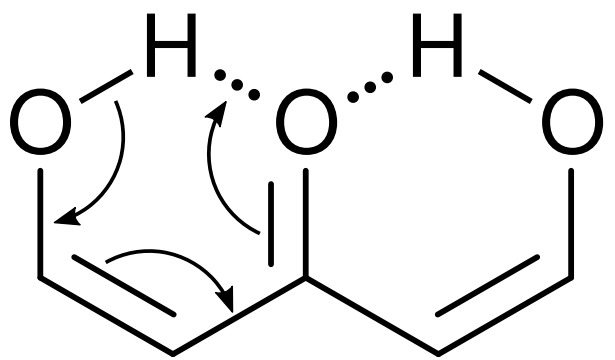

(d) 4

Figure 5: Resonance structures of the systems under investigation consistent with the H-bond cooperativity in system 2 and the anticooperative effects in compounds 3 and $\mathbf{4}$. We also show the mesomeric structures corresponding to $\mathbf{1}$ as a reference.

other HB and in its absence as a measure of cooperativity in these systems:

$$
\begin{aligned}
\Delta E_{\text {coop }}(\mathbf{2} \mathbf{a}) & =\Delta E_{\text {form }}(\mathbf{2} \mathbf{a})-\Delta E_{\text {form }}^{\prime}(\mathbf{2} \mathbf{a}) \\
\Delta E_{\text {coop }}(\mathbf{2} \mathbf{e}) & =\Delta E_{\text {form }}(\mathbf{2} \mathbf{e})-\Delta E_{\text {form }}^{\prime}(\mathbf{2} \mathbf{e}) \\
\Delta E_{\text {coop }}(\mathbf{3}) & =\Delta E_{\text {form }}(\mathbf{3})-\Delta E_{\text {form }}^{\prime}(\mathbf{3}) \\
\Delta E_{\text {coop }}(4) & =\Delta E_{\text {form }}(4)-\Delta E_{\text {form }}^{\prime}(\mathbf{4})
\end{aligned}
$$

The more negative the value of $\Delta E_{\text {coop }}(\mathbf{N})$, wherein $\mathbf{N}$ denotes either of the H-bonds 2a, 2e, 3 and 4, the larger the $\pi$ cooperative effects for this interaction. The converse holds for positive values of $\Delta E_{\text {coop }}(\mathbf{N})$ and $\pi$ anticooperativity in the system. It is not difficult to show that $\Delta E_{\text {coop }}(\mathbf{2 a})=\Delta E_{\text {coop }}(\mathbf{2} \mathbf{e})$. Table 1 reports the values of $\Delta E_{\text {form }}(\mathbf{N}), \Delta E_{\text {form }}^{\prime}(\mathbf{N})$ and $\Delta E_{\text {coop }}(\mathbf{N})$ from which we indeed observe that the hydrogen bonds of molecule 2 present $\pi$ 
Table 1: Assessment of $\pi$-non additive effects using the values of $\Delta E_{\text {coop }}(\mathbf{N})$ with $\mathbf{N}=\mathbf{2 a}, \mathbf{2 e}, \mathbf{3}$ and $\mathbf{4}$ as defined in equations (16)-(19). The values are reported in $\mathrm{kcal} / \mathrm{mol}$.

\begin{tabular}{cccc}
\hline HB & $\Delta E_{\text {coop }}(\mathbf{N})$ & $\Delta E_{\text {form }}(\mathbf{N})$ & $\Delta E_{\text {form }}^{\prime}(\mathbf{N})$ \\
\hline $2 \mathrm{a}$ & -3.64 & -7.09 & -3.45 \\
$2 \mathrm{e}$ & -3.64 & -15.72 & -12.08 \\
3 & 12.29 & -11.93 & -24.22 \\
4 & 1.84 & -12.79 & -14.63 \\
\hline
\end{tabular}

cooperative hydrogen bonds whereas molecules 3 and $\mathbf{4}$ exhibit HB $\pi$ anticooperativity. We highlight the positive value of $\Delta E_{\text {coop }}(3)=12.29 \mathrm{kcal} / \mathrm{mol}$ which is mainly due to the very large formation of one hydrogen bond of $\mathbf{3}$ in the abscence of the other HB in the molecule and also consistent with the proton transfer described in Figure 4.

The energy barriers for the dissociation in the plots of Figure 3 provide another way to characterize the $\pi$ non-additive effects of the systems examined in this work. As Figure 6 show, the activation energies for the dissociation of the H-bonds of molecule $\mathbf{2}$ are larger in the presence of the second HB, whereas the contrary is true for compounds $\mathbf{3}$ and $\mathbf{4}$. This means that the HBs in system 2 impair the dissociation of one another while the hydrogen bonds in $\mathbf{3}$ and $\mathbf{4}$ facilitate the breaking of any of the two interactions. This is consistent with the previous assignation of $\pi$ anticooperative effects in the molecules 3 and 4 along with $\pi$ cooperative effects in system 2 .

\subsection{Quantum chemical topological analyses}

Regarding the QTAIM and IQA analyses, first, we estimate the hydrogen bond formation energy by means of the topological properties of $\varrho(\mathbf{r})$, i.e., ${ }^{[45]}$

$$
E_{\mathrm{HB}}=\frac{1}{2} V\left(\mathbf{r}_{c}\right)
$$

wherein $V\left(\mathbf{r}_{c}\right)$ is the potential energy density at the bond critical point related to the $\mathrm{H}$ bond. Table 2 reports the values of $E_{\mathrm{HB}}$ together with those of the delocalisation index for the atoms directly involved in the hydrogen bond and the IQA interaction energy for the $\mathrm{O}-\mathrm{H}$ and $\mathrm{O} \cdots \mathrm{H}$ bonds in addition to their classical and exchange-correlation components. Despite the correspondence between the data in Table $2\left(\left|E_{\mathrm{HB}}\right|\right.$ and $\left|E_{\mathrm{int}}^{\mathrm{O} \cdots \mathrm{H}}\right|$ increase with $\delta(\mathrm{O} \cdots \mathrm{H})$ and diminish with $\delta(\mathrm{O}-\mathrm{H}))$, there is not a complete agreement of the cooperative and anticooperative effects predicted by Figure 3 and Table 2. For example, this chart predicts that the enolic hydrogen bond exerts a slightly anticooperative effect on the carboxylic acid 


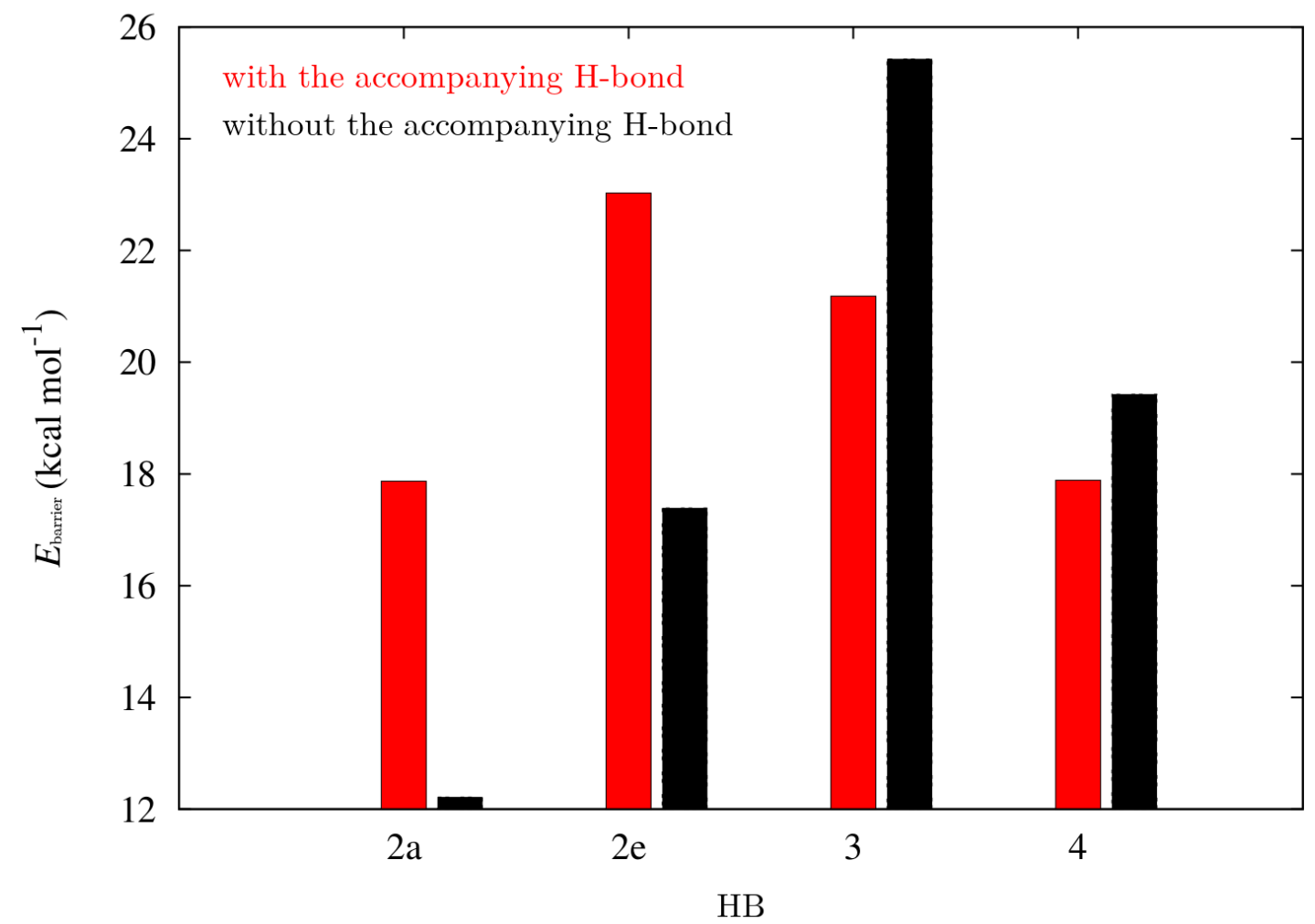

Figure 6: Potential energy barriers in systems 2-4 in the dissociation of the resonance assisted hydrogen bonds in Figure 2.

H-bond within compound $\mathbf{2}$ and that the RAHBs in system $\mathbf{3}$ strenghten each other, i.e., present cooperative effects.

We stress, however, that the topological values of Table 2 refer only to the atoms directly involved in the H-bond. Table 2 of reference [22] shows that the IQA interaction energies of the atoms that do not participate directly in the HB are also important in evaluating the formation energy of hydrogen bonding in opposition to the consideration of solely the properties in the $\mathrm{O} \cdots \mathrm{H}-\mathrm{O}$ region as done in expression (20). In the case of intermolecular interactions, say between two monomers $\mathscr{G}$ and $\mathscr{H}$, we can consider directly all pairwise values of $E_{\text {int }}^{\mathrm{A} \ldots \mathrm{B}}$ wherein atom $A$ belongs to monomer $\mathscr{G}$ and $\mathrm{B}$ to $\mathscr{H}$. This division is not straightforward in intramolecular interactions, but such an issue is elegantly addressed by IQA additive energies (expression (4)), to consider the effect of the hydrocarbon chain in the RAHBs under examination as reported in Table 3. The data show that the formation of the RAHB involves a stabilization of the atoms participating directly in the H-bond, but in addition, the energy of the rest of the system is substantially increased. This indicates that the hydrocarbon chain has an important effect in the RAHB formation energy and its different components. Indeed, the energetic change of the $\pi$ system modulates the magnitude of $\Delta E_{\text {form }}^{\mathrm{RAHB}}$. This complicates the quantitative establishment of the magnitudes of H-bond 
Table 2: Formation energies for the resonance assisted H-bonds shown in Figure 2 estimated through expression (20). The delocalisation indices $\delta(\mathrm{O} \cdots \mathrm{H})$ and $\delta(\mathrm{O}-\mathrm{H})$ together with the IQA interaction energies $E_{\text {int }}^{\mathrm{O} \cdots \mathrm{H}}$, $V_{\mathrm{cl}}^{\mathrm{O} \cdots \mathrm{H}}$ and $V_{\mathrm{x}}^{\mathrm{O} \cdots \mathrm{H}} \cdot E_{\mathrm{HB}}$ is reported in $\mathrm{kcal} / \mathrm{mol}$ whereas the rest of quantities are given in atomic units.

\begin{tabular}{lcccccc}
\hline RAHB & $E_{\mathrm{HB}}$ & $\delta(\mathrm{O} \cdots \mathrm{H})$ & $\delta(\mathrm{O}-\mathrm{H})$ & $E_{\mathrm{int}}^{\mathrm{O} \cdots \mathrm{H}}$ & $V_{\mathrm{cl}}^{\mathrm{O} \cdots \mathrm{H}}$ & $V_{\mathrm{x}}^{\mathrm{O} \cdots \mathrm{H}}$ \\
\hline 1 & -21.262 & 0.112 & 0.375 & -0.329 & -0.306 & -0.023 \\
2a (2e present) & -14.497 & 0.092 & 0.399 & -0.301 & -0.283 & -0.018 \\
2a (2e absent) & -14.865 & 0.091 & 0.412 & -0.296 & -0.278 & -0.018 \\
2e (2a present) & -23.367 & 0.119 & 0.358 & -0.354 & -0.328 & -0.026 \\
2e (2a absent) & -19.062 & 0.102 & 0.380 & -0.332 & -0.311 & -0.021 \\
3 (accompanying HB present) & -25.132 & 0.127 & 0.347 & -0.356 & -0.329 & -0.027 \\
3 (accompanying HB absent) & -32.039 & 0.142 & 0.325 & -0.383 & -0.351 & -0.032 \\
4 (accompanying HB present) & -18.105 & 0.102 & 0.388 & -0.324 & -0.304 & -0.020 \\
4 (accompanying HB absent) & -19.453 & 0.110 & 0.381 & -0.326 & -0.304 & -0.022 \\
\hline
\end{tabular}

formation energies in these molecules by the consideration of only the $\mathrm{O} \cdots \mathrm{H}-\mathrm{O}$ moiety as done in formula (20). Conversely, the hydrocarbon chain suffers important modifications on account of the RAHB in agreement with the notion that an HB may substantially affect the energy of a $\pi$-system ${ }^{[46]}$. More specifically to the non-additive effects previously described, the presence of either of RAHBs $\mathbf{2 a}$ or $\mathbf{2 e}$ is accompanied by larger magnitudes for $\Delta E_{\text {add }}^{\mathrm{O}-\mathrm{H} \cdots \mathrm{O}}$ and $\Delta E_{\text {add }}^{\cdots \mathrm{CH}=\mathrm{CH}-\mathrm{C} \cdots}$ for the formation of the other H-bond. The effect of the former being dominant leading thereby to the hydrogen bond $\pi$ cooperativity observed in molecule $\mathbf{2}$. A similar analysis of molecules $\mathbf{3}$ and $\mathbf{4}$, shows that the presence of a previous hydrogen bond reduces the stability of the $\mathrm{O}-\mathrm{H} \cdots \mathrm{O}$ system in consistency with the RAHB $\pi$ anticooperativity previously described for these molecules.

The quantities (16)-(19) can also be dissected in IQA net and interatomic contributions,

$$
\Delta E_{\text {coop }}(\mathbf{M})=\sum_{\mathrm{A}} \Delta E_{\mathrm{net} / \mathrm{coop}}^{\mathrm{A}}(\mathbf{M})+\frac{1}{2} \sum_{\mathrm{A} \neq \mathrm{B}} \Delta E_{\mathrm{int} / \mathrm{coop}}^{\mathrm{AB}}(\mathbf{M})
$$

wherein $\mathbf{M}=\mathbf{2}, \mathbf{3}$ or $\mathbf{4}^{1}$. A negative component of either $\Delta E_{\text {net/coop }}^{\mathrm{A}}(\mathbf{M})$ or $\Delta E_{\mathrm{int} / \mathrm{coop}}^{\mathrm{AB}}(\mathbf{M})$ is indicative of cooperativity of the H-bonds in species $\mathbf{M}$ and conversely for positive values of these quantities and anticooperativity. Figure 7 plots the values of $\Delta E_{\text {int/coop }}^{\mathrm{AB}}(\mathbf{M})$ for the $\mathrm{O} \cdots \mathrm{H}$ bonds and the covalent interactions throughout the pseudo-bicyclic structure of

\footnotetext{
${ }^{1}$ We recall at this point that $\Delta E_{\text {coop }}(\mathbf{2 a})=\Delta E_{\text {coop }}(\mathbf{2 e})$ as defined in equations (16) and (17).
} 
Table 3: Change in the IQA additive energies after the formation of the hydrogen bonds under examination in presence and abscence of the accompanying hydrogen bond within the molecule. The data are reported in $\mathrm{kcal} / \mathrm{mol}$.

\begin{tabular}{lccr}
\hline HB & $\Delta E_{\text {add }}^{\mathrm{O}-\mathrm{H} \cdots \mathrm{O}}$ & $\Delta E_{\text {add }}^{\cdots \mathrm{CH}=\mathrm{CH}-\mathrm{C} \cdots}$ & $\Delta E$ \\
\hline 1 & -22.27 & 12.99 & -9.28 \\
2a (2e present) & -20.33 & 16.38 & -3.96 \\
2a (2e absent) & -15.48 & 13.29 & -2.19 \\
2e (2a present) & -24.25 & 11.10 & -13.15 \\
2e (2a absent) & -20.64 & 9.25 & -11.38 \\
3 (accompanying HB present) & -21.86 & 12.91 & -8.96 \\
3 (accompanying HB absent) & -28.43 & 7.62 & -20.80 \\
4 (accompanying HB present) & -18.21 & 6.74 & -11.47 \\
4 (accompanying HB absent) & -20.93 & 9.27 & -11.66 \\
\hline
\end{tabular}

the compounds examined in this investigation. We observe that the value of $\Delta E_{\text {coop }}^{\mathrm{O} \cdots \mathrm{H}}(\mathbf{M})$ correspond with the cooperative $\left(\Delta E_{\text {coop } / \text { int }}^{\mathrm{O} \cdots \mathrm{H}}(\mathbf{2})<0\right)$ or anticooperative $\left(\Delta E_{\text {coop } / \text { int }}^{\mathrm{O} \cdots \mathrm{H}}(\mathbf{3})<0\right.$ and $\left.\Delta E_{\text {coop } / \text { int }}^{\mathrm{O} \cdots \mathrm{H}}(4)<0\right)$ effects discussed above. In correspondence with previous descriptions of other $\mathrm{RAHBs}{ }^{[39]}$ in which $V_{\mathrm{cl}}^{\mathrm{AB}}$ has a preponderant role in this interaction, the classical contribution is the dominant component of $\Delta E_{\text {coop/int }}(\mathbf{M})$. Appart from compound 4 , whose graph in Figure 7 strongly indicates anticooperativity of the H-bonds in this system (most of the shown bars are above zero), there is an alternating pattern of the data in Figure 7 concerning systems $\mathbf{2}$ and 3. Nonetheless, most of the bonds in the pseudo-bicyclic moiety of system 2 indicates cooperativity as shown in Figure 8-2. The opposite occurs in systems 3 and 4 (Figures 8-3 and 8-4). In other words, the $\pi$ cooperative effects of HB analyzed in this work are reflected in a more noticeable strengthening ${ }^{2}$ of the conjugated system governed by the classical interaction as shown in Table 4. A less fortified structure is related to the anticooperativity of RAHBs in systems $\mathbf{3}$ and $\mathbf{4}$, with larger anticooperative effects for the former molecule as mentioned above. As might be expected, the other contributions to $\Delta E_{\text {coop }}(\mathbf{M})$, i.e., $\sum_{\mathrm{A}} \Delta E_{\text {net } / \text { coop }}^{\mathrm{A}}(\mathbf{M})$ and $\frac{1}{2} \sum_{\mathrm{A} \neq \mathrm{B} \notin \text { bicyclic }} \Delta E_{\text {int } / \text { coop }}^{\mathrm{AB}}(\mathbf{M})$, modulate these cooperative (molecule $\mathbf{2}$ ) and anticooperative (systems $\mathbf{3}$ and $\mathbf{4}$ ) effects described above (Table S2 in the

\footnotetext{
${ }^{2}$ The formation of one or two HBs in molecules $\mathbf{2}, \mathbf{3}$ and $\mathbf{4}$ fortifies the bicyclic structures of these systems with respect to the system with no H-bonds as shown in Figures S3-S4 and Table S1 in the SI. Such strengthening is modified by the presence of one or two HBs in accordance with the hydrogen bond $\pi$ cooperative or anticooperative effects discussed in the paper.
} 
Table 4: Interaction energy components of $\Delta E_{\mathrm{int} / \mathrm{coop}}^{\mathrm{AB}}$ (equation (21)) across the hydrogen and covalent bonds forming the pseudo-byciclic moiety of compounds $\mathbf{2}, \mathbf{3}$ and $\mathbf{4}$. The data are reported in $\mathrm{kcal} / \mathrm{mol}$.

\begin{tabular}{lccc}
\hline System & $\sum_{\text {bicyclic }} \Delta E_{\text {int/coop }}^{\mathrm{AB}}$ & $\sum_{\text {bicyclic }} \Delta V_{\text {cl/coop }}^{\mathrm{AB}}$ & $\sum_{\text {bicyclic }} \Delta V_{\text {xc/coop }}^{\mathrm{AB}}$ \\
\hline $\mathbf{2}$ & -10.70 & -7.48 & -3.22 \\
$\mathbf{3}$ & 64.66 & 85.98 & -21.31 \\
$\mathbf{4}$ & 26.39 & 27.77 & -1.38 \\
\hline
\end{tabular}

SI).

The non-additive effects of the RAHBs considered in this study can also be analysed in terms of the sum of the intra and intermolecular exchange-correlation contributions to the total energy of the system,

$$
\begin{aligned}
& V_{\mathrm{xc}, \text { intra }}=\sum_{\mathrm{A}} V_{\mathrm{xc}}^{\mathrm{A}} \text { and } \\
& V_{\mathrm{xc}, \text { inter }}=\frac{1}{2} \sum_{\mathrm{A} \neq \mathrm{B}} V_{\mathrm{xc}}^{\mathrm{AB}},
\end{aligned}
$$

with a similar contribution to RAHB cooperativity or anticoopeartivity as in equation (21),

$$
\Delta V_{\mathrm{xc}, \mathrm{x} / \text { coop }}(\mathbf{N})=\Delta V_{\mathrm{xc}, \mathrm{x}}(\mathbf{N})-\Delta V_{\mathrm{xc}, \mathrm{x}}^{\prime}(\mathbf{N}),
$$

wherein $\mathrm{X}=$ intra or inter; $\mathbf{N}=\mathbf{2}, \mathbf{3}$, or $\mathbf{4}$ and the unprimed values refer to processes

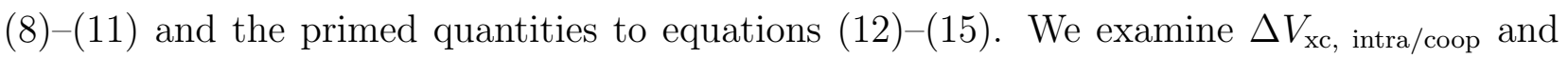
$\Delta V_{\mathrm{xc}}$, intra/coop because the formation of an RAHB is accompanied by a reduction of the magnitude of $V_{\mathrm{xc}}$, inter, at the expense of an increase of $\mid V_{\mathrm{xc}}$, intra $\left.\right|^{[39]}$. Table 5 shows that the components of $V_{\mathrm{xc}}$, intra and $V_{\mathrm{xc}}$, inter to $\Delta E_{\mathrm{coop}}(\mathbf{N})$ have a similar role in the previous description of RAHBs, i.e., the H-bonds in 2 rise the magnitude of $V_{\mathrm{xc}}$, intra and diminish that

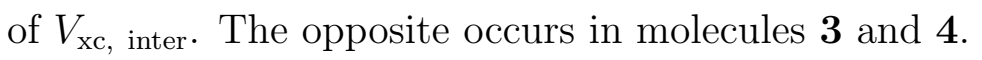

The changes in the quantities $V_{\mathrm{xc}}$, intra and $V_{\mathrm{xc}}$, inter in the formation of an RAHB are mirrored by those of the localised and delocalised electrons. Table S3 of the SI shows that the formation of every of the examined RAHBs conduces to a localisation of the electrons in the system. We can also compare the disminution of the delocalisation in a similar way to equations (21) and (24),

$$
\Delta \delta_{\text {coop }}(\mathbf{N})=\frac{1}{2} \sum_{\mathrm{A} \neq \mathrm{B}}\left(\Delta \delta^{\mathrm{AB}}(\mathbf{N})-\Delta \delta^{\mathrm{AB}}(\mathbf{N})\right), \mathbf{N}=\mathbf{2}, \mathbf{3}, \text { or } 4
$$


Table 5: Contributions of the intra and interatomic exchange components (in kcal $/ \mathrm{mol}$ ) to $\Delta E_{\text {coop }}(\mathbf{N})$ with $\mathbf{N}=\mathbf{2}, \mathbf{3}$, or $\mathbf{4}$ (equation (24)). The values of $\Delta \delta_{\text {coop }}$ (expression (25)) are reported as well.

\begin{tabular}{cccc}
\hline System & $\Delta V_{\mathrm{xc}, \text { intra/coop }}$ & $\Delta V_{\mathrm{xc}, \text { inter/coop }}$ & $\Delta \delta_{\text {coop }}$ \\
\hline $\mathbf{2}$ & -1.51 & 0.91 & -0.03 \\
$\mathbf{3}$ & 34.89 & -30.00 & 0.10 \\
$\mathbf{4}$ & 1.26 & -1.70 & 0.01 \\
\hline
\end{tabular}

in which the primed and unprimed quantities have the same meaning that in equation (24). We observe in Table 5 that the second H-bond in $\mathbf{2}$ leads to a larger diminution of delocalisation $\left(\Delta \delta_{\text {coop }}(\mathbf{2})<0\right)$ in contrast to 3 and $\mathbf{4}\left(\Delta \delta_{\text {coop }}(\mathbf{3})>0\right.$ and $\left.\Delta \delta_{\text {coop }}(\mathbf{4})>0\right)$. In other words, the results of Table 5 indicate that the cooperative/anticooperative RAHB effects studied herein reinforce/debilitate the electron localisation associated with this type on interaction ${ }^{[39]}$. These changes in the LIs and DIs correspond to those of the intra and interatomic changes in the components of the exchange-correlation to $\Delta E_{\text {form }}$ of a given resonance assisted hydrogen bond. In other words, the cooperative and anticooperative effects of RAHBs considered in this investigation can be inferred from the mesomeric structures of Figure 5. RAHB nonadditivy (system 2) follows from deviations of electron localisation as compared with an isolated resonance assisted hydrogen bond, which in turn modifies the values of $V_{\mathrm{xc}}$, intra, $V_{\mathrm{xc}}$, inter and above all $V_{\mathrm{cl}}$ which ultimately affects the strengthening of the pseudo-bicyclic structure associated to these interactions.

Finally, we discuss the changes in the electron delocalisation throughout the pseudobyciclic structure of compounds 2,3 and 4 . The changes in the delocalisation indices in the conjugated system 1 due to the formation of the RAHB, indicate that the double bonds involving one or two carbon atoms reduce their number of shared electrons, whereas the single bonds increase their associated DIs. ${ }^{[39]}$ In other words, the delocalised electrons become more evenly distributed across the conjugated $\pi$ system. This occurs despite the fact that this increase in the uniformity in the number of delocalised electrons is accompanied by an increment of the IQA additive energy forming the hydrocarbon chain as revealed in Table 3. It is noteworthy that the $\delta(\mathrm{O}-\mathrm{H})$ index is substantially diminished due to the generation of the RAHB. We considered the effect of the generation of one and two hydrogen bonds on the delocalisation indices throughout the conjugated system of molecules 2-4 (Figure 9). Overall, the delocalisation indices computed when only one RAHB is formed are intermediate between (i) the values calculated if the two hydrogen bonds are present in the molecule and (ii) the corresponding data observed when the two RAHBs are dissociated. Again, the formation 

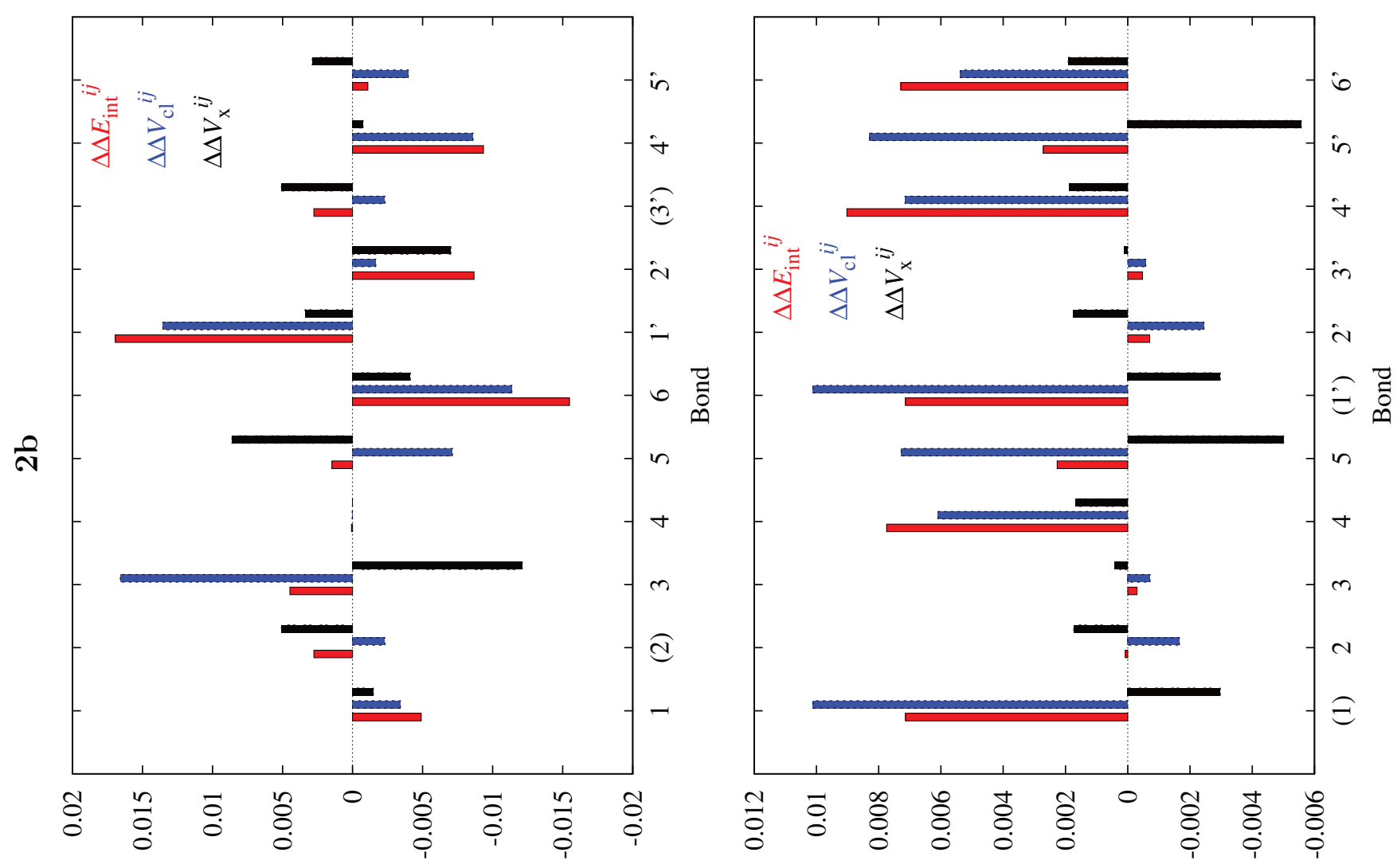

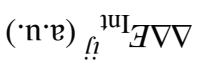

$(\cdot \mathrm{n} \cdot \mathfrak{e})_{? ! ?}^{1 \mathrm{UI}^{3}} \mathrm{I} \nabla \nabla$
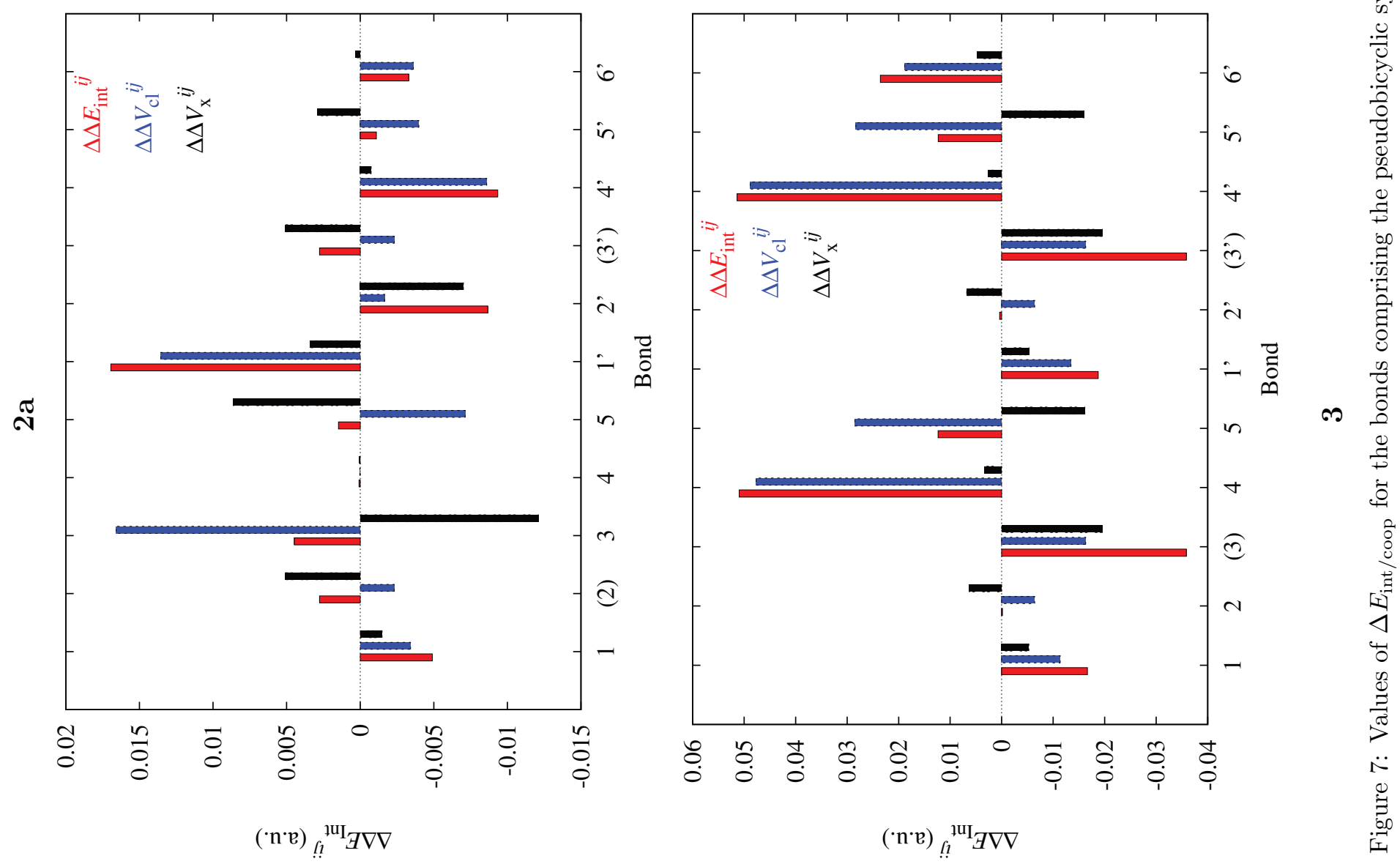


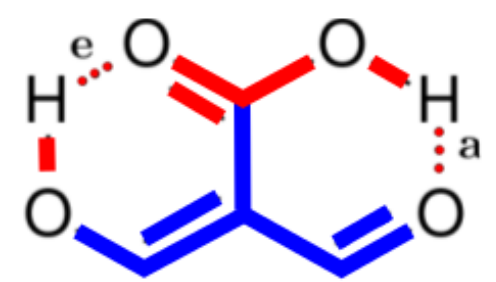

2

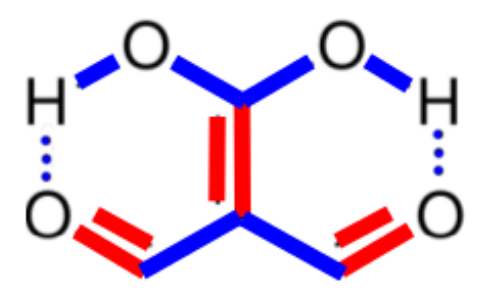

3

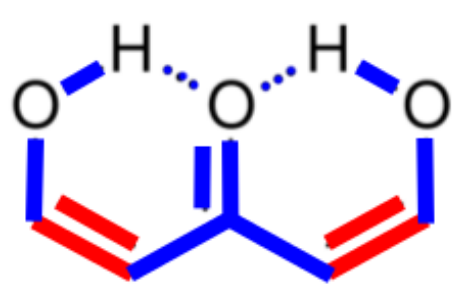

4

Figure 8: Cooperative (red) and anticooperative (blue) components of $\Delta E_{\mathrm{coop} / \text { int }}^{\mathrm{A}-\mathrm{B}}$ for contiguos atoms between the pseudo-bicyclic moieties for systems $\mathbf{2}, \mathbf{3}$ and $\mathbf{4}$.

of RAHBs, regardless of their cooperative or anticooperative nature, make the number of delocalised electrons across the system more uniform.

We have also analysed the alterations in the hydrocarbon chain in the systems of interest resulting from the formation of the examined RAHBs through the consideration of the change of the IQA energies in these processes. Figure S3 and S4 of the SI leads to similar conclusions than those based on delocalisation indices. When the RAHBs are formed, double bonds $\mathrm{A}=\mathrm{B}$ diminish their corresponding magnitude of $\left|E_{\text {int }}^{\mathrm{A}=\mathrm{B}}\right|$, whereas single bonds exhibit the opposite behaviour as revealed in the alternating patterns of the plots in Figures S3 and S4. Generally, the change in $\left|E_{\mathrm{int}}^{\mathrm{AB}}\right|$ for bonds which involve an oxygen atom are principally driven by $V_{\mathrm{cl}}^{\mathrm{AB}}$ while $V_{\mathrm{xc}}^{\mathrm{AB}}$ becomes more important for the bonds entailing only carbon atoms. The changes in the classical component are more important than those of the exchange component in accordance with a previous description of RAHB based on quantum chemical topology. [39]

\section{Concluding remarks}

We have presented an analysis of H-bond cooperative and anticooperative effects in $\beta$ enolones which result from the condensation of two molecules of malondialdehyde. For this, we compared the HB formation energies of the addressed resonance assisted hydrogen bonds with and without the accompanying H-bond in the corresponding molecules. The mesomeric structures of these systems are in agreement with the observed cooperative or anticooperative effects in the investigated HBs, which are also evidenced by the IQA interaction energies for the $\mathrm{O} \cdots \mathrm{H}$ bond. The modification of $E_{\text {int }}^{\mathrm{O} \cdots \mathrm{H}}$ on account of an accompanying H-bond is however not the only IQA component to be considered for a proper description of $\Delta E_{\text {form }}^{\mathrm{RAHB}}$. 

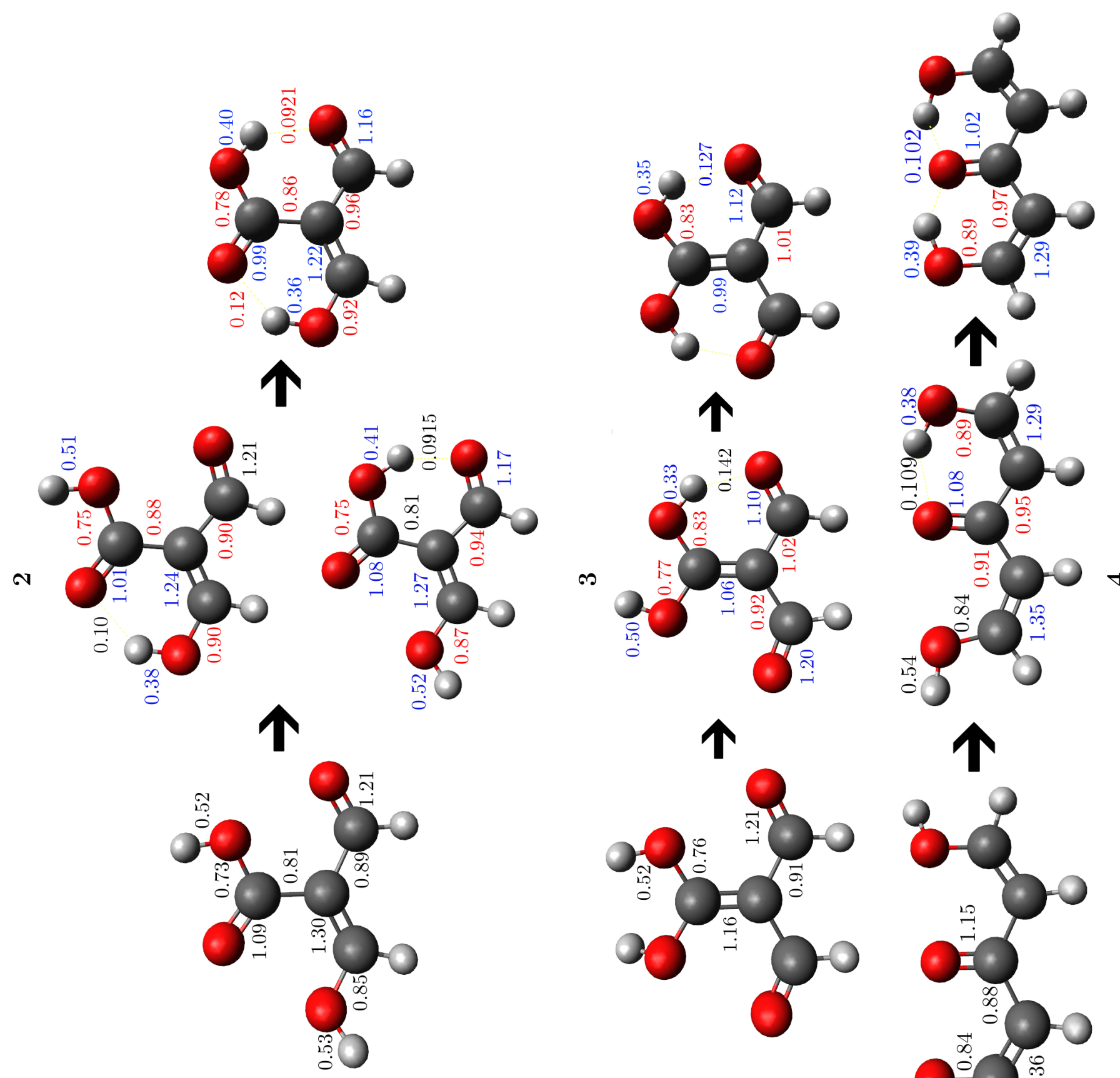

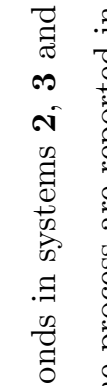


Indeed, the changes in the hydrocarbon chain are important in the establishment of the values of $\Delta E_{\text {form }}(\mathbf{N})$ and $\Delta E_{\text {form }}^{\prime}(\mathbf{N})$ with $\mathbf{N}=\mathbf{2 a}, \mathbf{2 e}, \mathbf{3}$ and $\mathbf{4}$ (equations (8)-(15)). Additionally, the IQA decomposition of $\Delta E_{\text {coop }}(\mathbf{M})$ wherein $\mathbf{M}=\mathbf{2}, \mathbf{3}$, or4 (expression (21)) shows that the pseudo-bicyclic structure of system $\mathbf{2}$ is more greatly strenghtened when the two H-bonds $\mathbf{2 a}$ and $\mathbf{2 e}$ are present as opposed to molecules $\mathbf{3}$ and $\mathbf{4}$ in which the same structure is stronger when only one HB is formed. Although the studied H-bond cooperative or anticooperative effects are mainly governed by the IQA classical contribution, the intra and intermolecular contributions of the exchange-correlation component of the RAHB formation energy are indicative of non-additivity in the examined H-bonds. Overall, we anticipate that the insights

presented in this paper will prove useful in getting a deeper understanding of in the interplay of hydrogen bonds and the functional groups surrounding these interactions.

\section{References}

[1] W. M. Latimer, R. W. H., J. Am. Chem. Soc. 1920, 42, 1419-1433.

[2] G. Gilli, P. Gilli, Int. Un. Cryst. Mon. Cryst.: The Nature of the Hydrogen Bond: Outline of a Comprehensive Hydrogen Bond Theory, Oxford University Press, USA, 2009.

[3] T. Steiner, Angew. Chem. Int. Ed. 2002, 41, 48-76.

[4] G. Gilli, P. Gilli, J. Mol. Struct. 2000, 552, 1-15.

[5] Unspecified author: So Much More to Know..., Science 2005, 309, 78-102.

[6] J. Trylska, P. Grochowski, J. McCammon, Protein Sci 2004, 13, 513-528.

[7] A. J. Stone, The Theory of intermolecular forces, Oxford University Press, New York, 1997.

[8] S. Scheiner, Hydrogen Bonding: A Theoretical Perspective, Oxford University Press, USA, 1997.

[9] G. Gilli, F. Bellucci, V. Ferretti, V. Bertolasi, J. Am. Chem. Soc. 1989, 111, 1023-1028.

[10] I. Alkorta, J. Elguero, O. M??, M. Y????ez, J. E. D. Bene, Chem. Phys. Lett. 2005, 411, 411-415.

[11] F. Fuster, S. J. Grabowski, The journal of physical chemistry. A 2011, 115, 10078-86.

[12] T. Cristina, G. Sánchez-Sanz, I. Alkorta, J. Elguero, O. Mó, M. Yáñez, J. Mol. Struct. 2013, 1048, $138-151$.

[13] J. Chin, C. K. Dong, H. J. Kim, F. B. Panosyan, M. K. Kwan, Organic Letters 2004, 6, 2591-2593.

[14] M. Rospenk, P. Majewska, B. Czarnik-Matusewicz, L. Sobczyk, Chem. Phys. 2006, 326, 458-464.

[15] C. Fonseca Guerra, F. M. Bickelhaupt, J. G. Snijders, E. J. Baerends, Chem. Eur. J. 1999, 5, 3581-3594. 
[16] C. F. Guerra, F. M. Bickelhaupt, J. G. Snijders, E. J. Baerends, J. Am. Chem. Soc. 2000, 122, 4117-4128.

[17] Y. Mo, J. Mol. Model. 2006, 12, 665-672.

[18] V. Bertolasi, L. Pretto, G. Gilli, P. Gilli, Acta Crystallogr. Sect. B-Struct. 2006, 62, 850-862.

[19] V. Bertolasi, P. Gilli, V. Ferretti, G. Gilli, J. Am. Chem. Soc. 1991, 113, 4917-4925.

[20] P. Gilli, V. Bertolasi, L. Pretto, A. Lycka, G. Gilli, J. Am. Chem. Soc. 2002, 124, 13554-13567.

[21] R. Otero, M. Schöck, L. M. Molina, E. Løgsgaard, I. Stensgaard, B. Hammer, F. Besenbacher, Angewandte Chemie - International Edition 2005, 44, 2270-2275.

[22] J. M. Guevara-Vela, R. Chávez-Calvillo, M. García-Revilla, J. Hernández-Trujillo, O. Christiansen, E. Francisco, A. Martín Pendás, T. Rocha-Rinza, Chem. Eur. J. 2013, 19, 14304-14315.

[23] J. M. Guevara-Vela, E. Romero-Montalvo, V. A. Mora Gomez, R. Chavez-Calvillo, M. Garcia-Revilla, E. Francisco, A. Martín Pendás, T. Rocha-Rinza, Phys. Chem. Chem. Phys. 2016,--

[24] D. Tiana, E. Francisco, M. A. Blanco, P. Macchi, A. Sironi, A. Martín Pendás, Phys. Chem. Chem. Phys. 2011, 13, 5068-77.

[25] D. Ferro-Costas, E. Francisco, A. M. Pendas, R. A. Mosquera, Phys. Chem. Chem. Phys. 2015, 17, 26059-26071.

[26] D. Ferro-Costas, A. M. Pendás, L. González, R. A. Mosquera, A. M. Pendas, L. Gonzalez, Phys. Chem. Chem. Phys. 2014, 16, 9249-9258.

[27] R. F. W. Bader, Atoms in molecules: A Quantum Theory, Oxford University Press, 1990.

[28] T. Helgaker, P. Jørgensen, J. Olsen, Molecular Electronic Structure theory, John Wiley \& Sons, Sussex, England, 2004.

[29] X. Fradera, M. A. Austen, R. F. W. Bader, J. Phys. Chem. A 1999, 103, 304-314.

[30] L. Albrecht, R. J. Boyd, O. Mó, M. Yáñez, Phys. Chem. Chem. Phys. 2012, 14, 14540.

[31] S. J. Grabowski, J. Phys. Org. Chem. 2003, 16, 797-802.

[32] R. W. Gora, S. J. Grabowski, J. Leszczynski, J. Phys. Chem. A 2005, 109, 6397-6405.

[33] C. Møller, M. S. Plesset, Phys. Rev. 1934, 46, 618-622.

[34] S. Kossmann, F. Neese, J. Chem. Theory Comput. 2010, 6, 2325-2338.

[35] T. H. Dunning, Jr., J. Chem. Phys. 1989, 90, 1007.

[36] F. Neese, Wiley Interdisciplinary Reviews: Computational Molecular Science 2012, 2, 73-78.

[37] C. Pérez, M. T. Muckle, D. P. Zaleski, N. A. Seifert, B. Temelso, G. C. Shields, Z. Kisiel, B. H. Pate, Science 2012, 336, 897-901. 
[38] M. W. Schmidt, K. K. Baldridge, J. A. Boatz, S. T. Elbert, M. S. Gordon, J. H. Jensen, S. Koseki, N. Matsunaga, K. A. Nguyen, S. Su, T. L. Windus, M. Dupuis, J. A. Montgomery, J. Comput. Chem. 1993, 14, 1347-1363.

[39] J. M. Guevara-Vela, E. Romero-Montalvo, , A. Costales, A. Martín Pendás, T. Rocha-Rinza 2016, Accepted for publication in Phys. Chem. Chem. Phys.

[40] T. A. Keith, AIMAll (Version 14.11.23), TK Gristmill Software, Overland Park KS, USA, 2012 (aim.tkgristmill.com).

[41] A. Martín Pendás, E. Francisco, Promolden. A QTAIM/IQA code (unpublished).

[42] T. Williams, C. Kelley, Gnuplot 4.6: An Interactive Plotting Program, 2010, http://gnuplot.sourceforge.net/.

[43] Avogadro: an open-source molecular builder and visualization tool. Version 1.XX. http://avogadro.openmolecules.net/.

[44] M. D. Hanwell, D. E. Curtis, D. C. Lonie, T. Vandermeersch, E. Zurek, G. R. Hutchison, J. Cheminf. 2012, 4:1\%.

[45] E. Espinosa, E. Molins, C. Lecomte, Chem. Phys. Lett. 1998, 285, 170-173.

[46] L. Gutierrez-Arzaluz, F. Cortes-Guzman, T. Rocha-Rinza, J. Peon, Phys. Chem. Chem. Phys. 2015, 17, 31608-31612. 